\title{
Carnets
}

Revue électronique d'études françaises de l'APEF

Deuxième série - 19 | 2020

Petite fabrique d'interprètes

\section{Giono : La Guerre, la Paix, L’Epidémie}

\section{Luis Carlos Pimenta Gonçalves}

\section{(2) OpenEdition}

\section{Journals}

Édition électronique

URL : http://journals.openedition.org/carnets/11472

DOI : 10.4000/carnets. 11472

ISSN : 1646-7698

Éditeur

APEF

\section{Référence électronique}

Luis Carlos Pimenta Gonçalves, «Giono : La Guerre, la Paix, L'Epidémie », Carnets [En ligne], Deuxième série - 19 | 2020, mis en ligne le 31 mai 2020, consulté le 23 décembre 2020. URL : http:// journals.openedition.org/carnets/11472; DOI : https://doi.org/10.4000/carnets.11472

Ce document a été généré automatiquement le 23 décembre 2020.

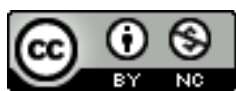

Carnets est mis à disposition selon les termes de la licence Creative Commons - Atribution - Pas d'utilisation commerciale 4.0 International. 


\title{
Giono : La Guerre, la Paix, L'Epidémie
}

\author{
Luis Carlos Pimenta Gonçalves
}

\section{NOTE DE L'AUTEUR}

Cet article reprend partiellement une communication sur les « Analogies et métaphores de l'épidémie chez Camus et Giono » lors du Colloque APEF « Albert Camus : cinquante ans plus tard ", qui s'est tenu à la Faculté des Lettres de l'Université de Lisbonne, le 1-2 octobre 2010, ainsi qu'une étude sur « La Grande Guerre à l'épreuve des textes et récits de Jean Giono ", publiée par Danièle Henky. 14/18 Dramatisation du récit de guerre. 2018. <hal-01808439>.

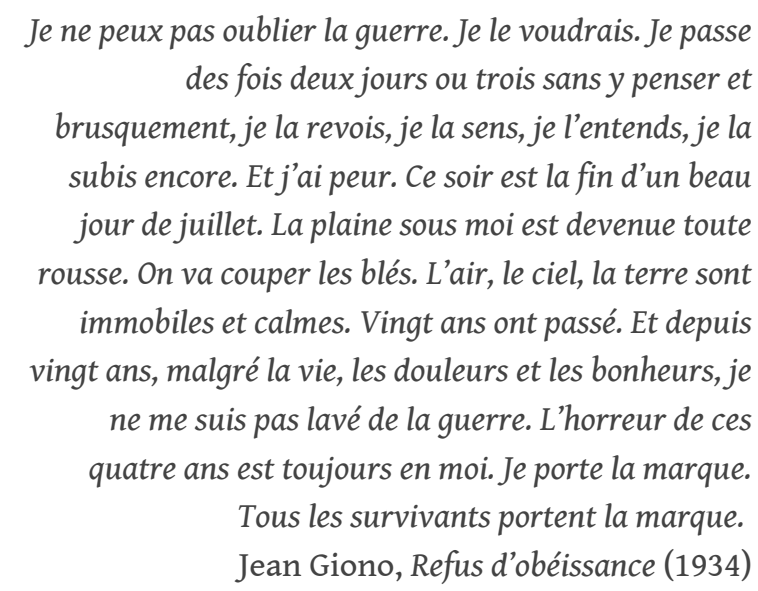

Dans les années 1980, alors jeune professeur à l'Alliance française de Beja, au sud du Portugal, figurait au programme du Diplôme Supérieur de Langue Française de l'Alliance française de Paris, un roman de Jean Giono, Le Hussard sur le toit. Ce fut la rencontre avec un auteur que je ne connaissais jusqu'alors qu'à travers les adaptations 
cinématographiques de Marcel Pagnol, fortement contestées par Giono, Regain ${ }^{1}$ à partir du roman éponyme et La Femme du boulanger ${ }^{2}$ tiré d'un épisode de Jean le Bleu.

Lecteurs et critiques ont longtemps voulu voir deux manières, deux styles, deux regards sur l'existence dans l'œuvre de Jean Giono33. Il y aurait, d'une part, une œuvre lumineuse, solaire, éminemment lyrique, orphique, panique sur les forces de la nature, la simplicité et la générosité d'hommes attachés à la terre vivant des «Vraies richesses » et, d'autre part, une autre œuvre, sombre, cruelle parfois où le meurtre devient alors le divertissement suprême, marquée par le pessimisme et le désabusement. Pour expliquer cette supposée césure entre ces deux façons d'écrire, il y la Seconde Guerre mondiale d'où Giono est sorti meurtri pour avoir été emprisonné pour pacifisme avant le conflit et accusé de collaboration après la Libération avant d'être libéré sans être inculpé4. Or, cette division en deux temps de l'œuvre romanesque de l'écrivain de Manosque est extrêmement simplificatrice, car c'est méconnaître ses quatre décennies de production littéraire de Naissance de l'Odyssée, achevé en 1927, à L'Iris de Suse, publié l'année de sa mort en 1970. Or, le caractère sombre nom exempt de cruauté et de rudesse commence tôt chez un auteur profondément bouleversé par la Première Guerre mondiale comme toute sa génération où il a perdu son ami intime Louis David en 1915, condisciple au collège de Manosque qui partageait ses «idées artistiques » (Giono, $1972: 1222)$ et ses aspirations.

Dans Refus d'obéissance, il déclare porter la marque de la guerre. C'est de cette marque inscrite, tout d'abord, dans sa correspondance de conscrit de la classe 1915, dans ses écrits pacifistes des années 1930, qui espéraient pouvoir contribuer à éviter un nouveau conflit dévastateur, dans ses romans comme Le Grand troupeau, qui se passe pendant la guerre de 14-18, dans Le Hussard sur le toit où la guerre surgit métaphoriquement en filigrane dans un récit qui parle d'une épidémie de choléra morbus dans la Provence de 1838, dont il sera question dans cette étude.

\section{La guerre vue des tranchées}

4 La publication de l'ensemble de la correspondance entre 1915 et 1919 de Jean Giono à ses parents ${ }^{5}$ permet de suivre son périple de soldat. La vision qu'il donne des combats est volontairement édulcorée et apaisante, elle est, de ce fait, bien plus anecdotique qu'épique. Les quelque cinq cents lettres conservées constituent un témoignage parmi tant d'autres de la Grande Guerre et permettent de découvrir un écrivain en devenir que l'épreuve a contribué à forger. À ce propos, Jacques Mény, dans sa " Présentation » du volume de la correspondance, estime que le lecteur est ici en présence d'un « récit de formation» (Giono, 2015: 6). L'objectif étant de rassurer les destinataires de ses lettres, Giono recourt fréquemment à des euphémismes. Il emploie humour désinvolte, persiflage et ironie, figures de style que nous retrouverons plus tard sous la plume de l'écrivain.

5 Ainsi, les longues marches forcées qui le mènent sur les lieux des combats sont interprétées comme formatrices : « les voyages forment la jeunesse et j'en retirerai des bénéfices considérables» (Giono, 2015: 7). De fait, son œuvre future est d'une certaine façon à mettre au compte d'un de ces "bénéfices ». Jacques Mény montre aussi que la découverte de la guerre par Giono est graduelle et non brutale comme on l'a d'abord cru. En effet, «Le jour même où Giono a rejoint les unités combattantes du $140^{\mathrm{e}} \mathrm{RI}$, le régiment vient d'être mis en repos pour trois semaines à Lavallée, petit village situé à 
quarante-cinq kilomètres au sud du front » (Giono, 2015 : 14). La première année de guerre nourrit encore l'espoir, partagé par beaucoup de soldats sur le front et l'opinion publique à l'arrière, que la fin des combats est proche comme il l'affirme à ses parents le 15 mars 1915: «La guerre sera bientôt finie, c'est un des derniers coups ». En est-il vraiment convaincu ou s'agit-il de cette stratégie qui vise à délivrer les siens de l'inquiétude? C'est, en tout cas, ce que laisse entendre un peu plus loin ses paroles: "écrivez-moi que vous ne vous faites pas du mauvais sang sur mon compte. Figurezvous que ma vie se passe à rire, à lire et jamais à être malheureux. » (Giono, $2015: 55$ ). Le jeune Giono insiste dans ses lettres à sa famille pendant les deux premières années de guerre sur ses espoirs d'être promu caporal puis d'arriver au grade de souslieutenant. Il évoque les exercices militaires et le temps libre dont il profite et qui lui permet de découvrir la région où il est cantonné. En 1916, les lettres se poursuivent avec la même teneur et sur le même ton. Le jeune conscrit ne semble être parti que pour réaliser un voyage d'agrément et de découverte. La vie semble bucolique et les parents de Giono doivent malgré tout être surpris d'apprendre que leur enfant passe une partie de son temps à cueillir des fraises dans les bois: "C'est tout à fait villégiature » précise-t-il (Giono, $2015: 122)$. Si son objectif premier n'était de dresser une fresque édulcorée et rassurante de la guerre, on pourrait le croire naïf tant il abuse d'appréciations lénifiantes. Il répète ainsi : « c'était très intéressant » (Giono, $2015: 123$ ) quand il assiste, par exemple, à un combat aérien. Il déclare : «on est très bien » (Giono, 2015: 127) alors qu'il se trouve en fait alité pour "courbature fébrile et anémie » comme il l'explique à ses parents le 28 juin 1916. Revenant sur son état de santé le jour suivant, il mentionne le transport en ambulance "confortablement couchés dans des automobiles américaines (...) On avait tout de Rothschild». Dans le train les malades et blessés sont couchés "comme des guerriers antiques sur des boucliers» (Giono, 2015: 127). Par ses comparaisons, l'écrivain relativise ses souffrances et manifeste également son goût déjà prononcé pour l'affabulation qui nourrira sa création future. Même si ces lettres n'ont rien de la correspondance d'un écrivain - qu'il n'est pas encore - on pressent en lisant un style qui recourt aux formules lapidaires et un imaginaire qui fictionne le réel, que nous sommes en présence d'un auteur en gestation.

6 La guerre elle-même est dévalorisée. Le pire ennemi du soldat dans les tranchées n'est pas l'Allemand que l'on combat, mais la multitude de parasites, d'insectes et de rongeurs. En faisant usage d'hyperboles, il esquisse un tableau où la réalité la plus repoussante devient pittoresque. Les poux sont "gros comme des petits chiens" (Giono, 2015 : 139), les rats "gros comme des petits lapins» (Giono, $2015: 156)$ ou encore les moustiques "gros comme des éléphants et épais comme des nuages " (Giono, $2015: 230$ ). Quand il lui arrive de faire part d'une nouvelle inquiétante, il ne peut s'empêcher d'en tirer une conséquence positive. Par exemple, le 23 août 1916, il annonce que «le régiment est décimé » (Giono, 2015 : 145) et précise aussitôt: «de longtemps nous ne pourrons plus rien faire. Nous voilà tranquilles ». Giono insiste sur la fin des périls toute proche quand son régiment s'éloigne de la zone du front: « $\mathrm{Ne}$ vous faites pas du mauvais sang. Ici on ne dirait plus qu'on est en guerre» (Giono, 2015 : 147-148). Même quand des obus tombent, la feinte insouciance du soldat continue de se manifester, car, écrit-il : «c'est de la 'Kamelote'« (Giono, 2015 : 162). Un obus allemand récupéré et détourné de sa fonction première trouve un usage bien plus pacifique et devient pour sa « petite maman un magnifique et paisible pot à fleurs» (Giono, 2015 : $170)^{6}$. 
7 Dans le quotidien du poilu la place de la lecture de livres ou de journaux est essentielle pour chasser l'ennui et encore plus pour le futur écrivain qui réclame à ses parents l'envoi de livres et raconte avec délectation l'existence d'une bibliothèque dans les environs. Chaque réception de colis de victuailles partagé entre soldats est considérée comme un moment de festivité en temps de guerre. Giono raconte ainsi qu'avec ses camarades, il a fait la «bombe » à diverses reprises, ou une "grande nouba » (Giono, 2015 : 173), ou encore, "Cette nuit, il y aura encore bamboula (Giono, $2015: 175)$. Giono, comme pour attester de sa bonne humeur et de son excellent moral, se risque même à faire des calembours en annonçant une permission : "J'espère vous trouver en bonne santé (...) et alors ce sera la bombe. Ici en fait de bombes, ça se pose un peu là, c'est du $210 »$ (Giono, 2015 : 224). L'optimisme du futur romancier est ainsi constant dans sa correspondance, il fait d'ailleurs allusion à Candide, dans une lettre du 27 mai 1918, en dédramatisant des nouvelles du front qui ont pu parvenir à sa famille : «Tout va bien après une petite échauffourée. Tout est pour le mieux dans le meilleur des mondes, comme disait ou contredisait Voltaire. » (Giono, $2015: 287$ )

8 Le récit qu'il fait de la guerre auprès des siens se retrouve dans un texte publié en 1961, Le Grand théâtre ${ }^{7}$. Il raconte avoir décrit brièvement pour son père, Jean-Antoine Giono, son expérience de la guerre lors d'une permission. Il le fait tout d'abord avec les précautions d'usage adoptées dans les lettres à sa famille qui visent à dédramatiser les risques et les souffrances encourus, puis en essayant de moins travestir le vécu et le ressenti.

(...) je lui décrivis avec beaucoup de détails notre vie dans la boue, nos sommeils souterrains, notre peur des espaces vides, notre besoin d'encoignures et de cachettes, l'étrange sensation quand nous nous tenions debout, l'éclatement des obus et ce sifflement précurseur qui nous aplatissait sur le sol. Je décrivais avec encore un peu plus de complaisance mon expérience de Verdun, mais en me bornant toutefois à parler des transformations constantes du paysage, du ciel, des lumières, des flammes, du bruit, des aveuglements, des assourdissements, de cette mise en pétrin et de ce brassage de terre et d'hommes, de cette absence totale de réalité qui en résultait. (Giono, $1974: 1087$ ).

\section{Le pacifisme de l'entre-deux-guerres}

9 À la seule lecture de sa correspondance avec sa famille où Giono inclut à diverses reprises, surtout à partir de 1917, des appréciations aux accents patriotiques sur la défaite et la mort de "Boches", on peut douter d'un réel pacifisme précoce chez l'écrivain provençal, tel que l'envisage généralement la critique. Pierre Citron présente ainsi Giono comme un « pacifiste viscéral depuis 1914 » (Citron, 1985 : 25). L'éditeur de Récits et essais de Jean Giono, volume publié dans la Bibliothèque de la Pléiade, nuance toutefois dans ce même texte son propos, car en «fabulateur viscéral» que le réel dérange, l'écrivain de Manosque recompose la réalité aussi bien dans ses œuvres de fictions que dans ses essais et dans son journal. Ainsi, le soldat de $2^{\text {e }}$ classe Jean Giono se présente volontiers comme le seul rescapé de la $6^{\mathrm{e}}$ compagnie du $140^{\mathrm{e}} \mathrm{R} . \mathrm{I}$ avec son capitaine, comme l'indique d'ailleurs son article pacifiste de 1934: "Je ne veux pas oublier », publié dans le numéro spécial de la revue Europe consacré à "1914-1918». Pourtant, selon Pierre Citron qui a consulté le Journal des Marches et Opérations du régiment conservé aux archives de l'armée de terre, les pertes n'auraient pas été aussi effroyables. La compagnie où sert Giono a été bien plus épargnée que d'autres, car son commandant, le capitaine Vidon ${ }^{8}$, a voulu préserver le plus possible la vie des soldats 
qui servaient sous ses ordres. Ce qui fait dire à sa veuve écrivant à Giono le 14 septembre 1935: " heureusement, Monsieur, vous ne restez pas seul survivant de la sixième du 140 (...) que de fois j'ai vu rentrer mon mari joyeux d'avoir rencontré un des anciens de sa 'belle sixième'" (Citron, $1985:$ 34). Dans cette recomposition du réel entre en ligne de compte un enjeu capital pour lui qui est de dénoncer, non seulement la guerre de 14-18, mais également le réveil des discours patriotiques et militaristes.

À la fin des années trente, son engagement pacifiste qui prône le désarmement immédiat de la France le conduit à rédiger un vigoureux pamphlet intitulé Précisions à partir de diverses interventions publiques et écrits de l'écrivain. Avant sa publication le 4 janvier 1939 pour les éditions Grasset, de larges extraits étaient déjà parus dans l'hebdomadaire pacifiste La Patrie humaine. Dans une adresse au président du conseil Édouard Daladier, il se décrit comme un des survivants des mutineries des soldats sur le front en 1917.

C'est un ancien soldat qui vous parle et cette gravité, monsieur le président, c'est la deuxième fois que je la vois sur des visages de soldats. La première fois je l'ai vue sur les visages de soldats de 1917. (...) J'ai fait partie des mutinés de cette époque, humblement. Je sais ce que c'est d'être aligné devant un adjudant qui compte : «1, $2,3,4$, le 4 sortez », et ainsi de suite, et tous les « $\mathrm{n}^{\circ} 4$ » sont fusillés le lendemain à l'aube, sans jugement. (Giono, 1989 : 609).

Or, rien ne vient corroborer cette affirmation et il s'agit plutôt d'une réécriture de l'histoire à vocation pamphlétaire dont le but est de convaincre l'opinion publique. Pour Pierre Citron, Giono «s'invente un passé de mutin de 1917 » (Citron, 1985 : 35) et donne comme preuve le fait que ce passé de révolté n'est revendiqué que tardivement auprès de sa famille, entre 1935 et 1939. Par ailleurs, s'il avait été véritablement témoin de ces exécutions, les mutineries ne seraient sûrement pas absentes de son roman Le Grand troupeau. Finalement, dernière preuve et non des moindres, le $140^{\mathrm{e}}$ Régiment d'Infanterie Alpine auquel est affecté Giono n'a pas connu de révoltes comme d'autres unités si l'on en croit le rédacteur du Journal des Marches et Opérations : «Au moment où l'armée française traverse une crise terrible, le régiment ne 'flanche' cependant pas et chacun sait rester digne et discipliné. » (Giono, $2015: 30$ ). La modification de données biographiques n'est pas uniquement due à l'imagination fertile de Giono ou à son désir de convaincre par ses pamphlets, mais découle également de la volonté de rechercher le sens profond des événements au-delà de la simple expérience personnelle. À ce titre, Katia Thomas Montésinos constate, dans un article sur «Jean Giono et la guerre de 14-18: une expérience tragique et féconde ", que, contrairement à ce que l'écrivain a affirmé dans " Je ne veux pas oublier » et dans Recherche de la pureté, il n'a pas participé en février-mars 1915 à l'assaut des Éparges puisqu'il était encore loin du front. Il a certes combattu avec son unité dans ce secteur, mais seulement en août 1916. Cette bataille s'inscrit dans un continuum mythifié d'épisodes guerriers, qui plongent dans la nuit des temps, et subliment une «absence " de réel ou plutôt d'un réel horrifique indescriptible.

Dans le numéro de juillet 1935 du journal d'Henri Barbusse, Le Monde, Giono réaffirme son pacifisme dans un texte intitulé "Certitude».

Si j'ai obéi la première fois, j'avais des excuses. Si j'obéissais à l'ordre d'une nouvelle guerre - n'importe laquelle - je serais à jamais déshonoré devant les générations futures, devant l'enchaînement de la vie dans le monde, devant ce qui existe, et devant ce qui, en moi-même, est immortel. ${ }^{9}$ 
13 La même année, dans Que ma joie demeure, l'écrivain propose une vision de la vie qui passe par la redécouverte du travail de la terre et du bonheur simple qui en découle et qui mène, selon une expression utilisée dans un avant-propos manuscrit, à « l'établissement de la joie ». Ce roman puis l'essai Les Vraies Richesses, publié en 1936, faisant un appel à un retour à la nature que l'on pourrait considérer comme étant une sorte d'écologie avant l'heure, vont amener à Manosque des groupes d'admirateurs. Le nombre est tel que Giono décide de les emmener en excursion sur la montagne de Lure en Haute-Provence. C'est lors d'une "promenade " à pied de quinze kilomètres, le $1^{\mathrm{er}}$ septembre 1935 (Giono, 1989 : 147), entre Manosque et le hameau du Contadour d'un groupe de plusieurs dizaines de personnes sur les lieux qui ont inspiré Giono que va naître ce qui deviendra de façon plus au moins informelle jusqu'en 1939 les Rencontres $\mathrm{du}$ Contadour $^{10}$. Il $\mathrm{y}$ aura en tout sept rencontres dont les thèmes de discussion alimenteront les huit numéros des Cahiers du Contadour, entre juillet 1936 et février 1939, où figureront des inédits et des textes de Giono, ainsi que d'autres auteurs. À partir du numéro double III-IV, du 25 septembre 1937, prédomineront les articles et sujets sur la paix et sur le pacifisme. L'écrivain Pierre Magnan (1922-2012), le plus jeune des Contadouriens, a quinze ans quand il débarque au Contadour en septembre 1937. Dans son livre de mémoires et d'amical hommage, Pour saluer Giono, il raconte les veillées où le romancier lit «Le vin qu'ils ont bu à l'aube » de Batailles dans la montagne qui vient de paraître. Magnan évoque également lors de ce séjour les conversations du groupe autour de l'attitude à adopter en cas de guerre: « renvoyer son fascicule de mobilisation, résister aux gendarmes, faire un fort Chabrol de la paix, se laisser fusiller sur place et pour les femmes se coucher sur les rails dans les gares. " (Magnan, 2002 : 54). On attend de Giono qu'il indique les consignes à suivre, ce qu'il se refuse à faire comme le soulignent le mémorialiste et ceux qui ont participé à ces discussions. Pour l'auteur de Jean le Bleu, "chacun devait agir selon sa propre conscience " (HellerGoldensberg, 1972: 152). Dans Les Cahiers du Contadour III-IV, l'écrivain donne un semblant de réponse jouant sur la provocation qui lui vaut alors bien des inimités et bien des déboires au sortir de la guerre : «Refuser d'obéir à la guerre. Que peut-il nous arriver de pire si l'Allemagne envahit la France? Devenir Allemands? Pour ma part, j'aime mieux être Allemand vivant que Français mort. »

\section{Fictionner l'expérience de la guerre}

Contrairement à d'autres écrivains qui, rentrés du front, livrent aussitôt leur vision terrifiante de la guerre - tel Henri Barbusse avec le Feu, Journal d'une escouade, paru en 1916 - Léon Werth avec cet autre réquisitoire contre la guerre qu'est Clavel soldat, publié à la sortie de la guerre en $1919^{11}$ ou encore Roland Dorgelès qui obtient la même année le Prix Femina pour Les Croix de bois, témoignage et pamphlet contre le militarisme, Giono attend bien plus longtemps avant de décrire un conflit qui a marqué profondément toute une génération. Le romancier de Manosque diffère l'écriture de ses années de guerre comme s'il fallait tout d'abord purger sa mémoire de souvenirs extrêmement violents accumulés pendant plus de deux années sur le front. Finalement, il trouve le moyen de le faire par le biais de la fiction. C'est d'abord le cas avec "Ivan Ivanovitch Kossiakoff ", nouvelle écrite en 1925, bien qu'elle soit incorrectement datée de 1920, qui intègre le recueil de Solitude de la pitié $e^{12}$. Jean Giono y raconte une histoire inspirée d'un fait réel, celle d'un soldat russe fusillé pour mutinerie avec lequel Giono - 
qui est aussi le narrateur du récit - s'était lié d'amitié. Mais c'est surtout dans Le Grand Troupeau, en 1931, qu'il trouve le moyen d'exprimer l'indicible dans un roman. Il lui aura fallu treize ans pour décrire les peurs et les angoisses qu'il a vécues. La découverte de l'effroi de la guerre par un des personnages est d'abord éminemment sensorielle et empreinte de la cruauté que l'on va retrouver dans les œuvres de maturité de l'auteur ${ }^{13}$. $\mathrm{Au}$ fond d'un trou à vingt mètres d'une mitrailleuse allemande le personnage se sent épié.

Il sentit comme une présence derrière lui. On le regardait. Il se retourna : c'était, sur l'autre bord du trou, un homme couché et qui avait la figure toute noire; sa cervelle coulait par une large blessure en coin. Il ne regardait pas ; c'était un petit morceau rond et blanc de cette cervelle qui faisait le regard parce qu'il était collé sur ce noir de l'œil, sur l'œil pourri et plein de boue. ${ }^{14}$

Cette fiction, au même titre que les écrits pacifistes de Giono, a en quelque sorte une valeur prophylactique à l'approche des périls qui vont déboucher sur la Seconde Guerre mondiale. Pourtant, il ne s'agit pas d'un simple acte de propagande écrit dans l'urgence. Le roman est extrêmement travaillé comme l'attestent ses brouillons dont le résultat final est expurgé de nombreux passages pour garder un certain équilibre de composition. Il se structure en trois parties dont les titres de certains chapitres sont autant d'allusions directes à L'Apocalypse ${ }^{15}$. Quatre des chapitres éliminés ou profondément remaniés dans la version définitive sont publiés plus tard dans son pamphlet de 1937, Refus d'obéissance: "Montée à Verdun ", "Veille d'attaque à SaintQuentin », "Quiconque donc me trouvera me tuera!», «Bataille du Kemmel». Ce dernier chapitre est une version moins travaillée, mais plus développée de l'avantdernier chapitre qui reprend le titre du roman : «Le Grand troupeau ». On y retrouve les mêmes descriptions, les mêmes personnages et dialogues, mais agencés de façon différente. Ainsi, dans le chapitre inédit «bataille du Kemmel», l'osmose entre les hommes et les lieux qu'ils traversent est rendue par une métaphore anthropomorphique : "Un village là-devant avec des maisons qui perdent leurs tripes de matelas et l'os brisé des meubles» (Giono, 1989: 309) qui devient de façon plus épurée dans la version finale du roman : « devant un gros village étripé et qui perd ses boyaux dans les champs» (Giono, 1971 : 705). Ailleurs, une comparaison qui introduit un élément dérisoire en déshumanisant des combattants: «Deux soldats anglais courent, penchés, les bras pendant comme des gros singes » (Giono, $1989: 310)$ devient plus sobrement : « Deux soldats anglais courent, les bras pendants » (Giono, 1971 : 706 ).

Dans les œuvres de maturité de l'écrivain sont également décelables les traces des deux guerres mondiales, parfois de façon diffuse sous forme de simple réminiscence ou d'analogie. En 1938, dans Les Cahiers du Contadour V, daté du 23 mai, Giono établissait dans un texte non signé une équivalence entre épidémie et guerre.

Dès qu'un choléra, une peste, une guerre de cent ans s'abat sur l'humanité, on voit se créer au milieu des tourbillons du malheur des sociétés d'honnêtes gens, sourds aux mots d'ordre, dédaigneux des habituels remèdes, qui continuent obstinément à chercher leur joie.

Ce parallélisme entre choléra et guerre, nous le retrouverons dans Le Hussard sur le toit, car Giono est fasciné par des phénomènes naturels ou provoqués par l'homme qui risquent de l'anéantir, le dépassent, lui révèlent ses grandeurs et ses misères. De ce fait, Pierre Citron dans sa notice pour la Bibliothèque de la Pléiade observe l'importance de l'expérience de la guerre dans l'écriture de trois œuvres majeures du romancier. 
Le souvenir du drame horrible de la guerre de 1914-1919 avait nourri Le Grand Troupeau. Le pressentiment de la guerre de 1939-1945 avait peut-être été l'une des origines de Batailles dans la montagne. Il est difficile de ne pas voir aussi dans ces deux guerres une des sources essentielles du Hussard sur le toit. (Citron, 1977 : 1307).

$18 \mathrm{Au}$ demeurant, n'oublions pas que le jeune Giono est contemporain de deux catastrophes qui s'interpénètrent : le premier conflit mondial et l'épidémie de la grippe espagnole qui en faisant des dizaines de millions de victimes, entre 1918 et 1919, a été plus meurtrière que la guerre qui s'achevait. Cette maladie par sa dévastation persiste dans la mémoire collective au même titre que les grandes épidémies de peste et de choléra des siècles précédents. Il n'est pas entièrement anodin, bien que Giono refuse de l'admettre, que le choléra constitue une allégorie de la guerre dans Le Hussard et que les deux finissent par se mélanger dans le récit. Après tout le personnage central, Angelo, est hussard, les villages infestés que traverse le héros sont dans un véritable état de siège avec des hommes en armes. Tout cela finit par constituer une isotopie sémantique commune. Pourtant, aucun commentaire et déclaration de Giono ne viennent étayer l'hypothèse qu'il faille directement établir un lien entre une épidémie qui se déroule en 1838 sous la monarchie de juillet et la guerre au vingtième siècle, et, a fortiori, à des épisodes de l'occupation et de la résistance. Dans un texte accompagnant en 1956 l'édition du Hussard sur le toit pour Le Club du Meilleur livre, Giono parle de son grand-père piémontais, carbonaro, camarade du père de Zola, qui l'aurait inspiré dans la composition du personnage du Hussard, Angelo. Il ajoute à propos de l'épidémie : «Quant au choléra (qui pourrait passer pour symbolique; cela s'est déjà vu), il a vraiment existé à cette époque, et aussi violent que je l'ai fait.»(Giono, 1977 : 1183) L'incise qui renvoie aux textes parlant de l'épidémie et peut-être également à La Peste de Camus, qu'il ne pouvait ignorer, ne récuse pas d'emblée l'interprétation analogique qu'il laisse aux exégètes en suspens.

19 Thomas-Montésinos dans l'article déjà cité fait un rapprochement biographique qui peut sembler hasardeux, mais qui demeure séduisant. Elle rappelle que l'écrivain, jeune mobilisé en 1916, a été affecté à un secteur surnommé Berry-Choléra et que la dysenterie que connaissent alors les soldats ressemble assez aux symptômes du choléra décrits par Giono dans son roman Le Hussard sur le toit. De même, dans le roman Le Grand troupeau les terribles descriptions de la guerre de 14-18 avec ses mourants et ses cadavres dévorés par les rats ressemblent étrangement aux victimes de l'épidémie, abandonnées dans les maisons et sur les routes de la Provence du Hussard. Au-delà de cette anecdote biographique qui se déroule pendant la Grande Guerre, le lecteur attentif pourra déceler dans le Hussard certains indices textuels qui renvoient de façon allusive à l'atmosphère que l'on pouvait vivre à la fin des années trente et pendant la Seconde Guerre. Le roman de Giono commence par la description d'Angelo et par les événements annonciateurs de l'épidémie de choléra qui ressemblent par leurs descriptions chronologiques à des procès-verbaux de gendarmerie. Ainsi au chapitre I, nous découvrons «Un médecin juif, alerté par un rabbin, surtout inquiet de pureté, vint examiner trois cadavres culbutés juste sur le seuil de la petite porte de la synagogue " (Giono, 1977: 253). Trois pages plus loin, ce praticien, craignant la contagion, enjoint à sa famille de partir.

C'était aussi exactement le moment où, le médecin juif étant rentré précipitamment chez lui, ayant parlé à sa femme, lui ayant fait préparer une petite valise pour elle et pour leur petite fille de douze ans, cette femme aux yeux de bœuf et à nez d'aigle quittait Carprentas par la diligence de Vaison avec l'ordre de pousser vite au-delà 
en voiture de louage jusqu'à Dieulefit et même jusqu'à Bordeaux. » (Giono, 1977 : 255-256). nord de Carpentras dont le nom est presque l'homophone et l'homographe de Bordeaux, ville d'où s'échappèrent vers l'exil des milliers de juifs français et allemands sous la menace nazi ${ }^{17}$, n'est pas sans rappeler celle de beaucoup de familles jetées sur les routes après la défaite de juin 1940. Le fait que la famille du médecin soit juive redouble le parallélisme entre le début d'une épidémie et les conséquences de la débâcle de l'armée française provoquant l'exode de la population, notamment juive. La militarisation de l'épidémie et l'organisation d'une résistante ne sont pas sans rappeler aussi celle qui se constitue pour faire face à l'occupant allemand et au régime de Vichy. Les bivouacs, les camps installés sur des collines aux environs de Manosque avec leurs ouvriers en armes ressemblent assez aux maquis des francs-tireurs et des partisans. Ainsi, au chapitre IX, pouvons-nous lire une description où l'organisation militaire se double d'une organisation civile.

[Ils] disaient leur mot paisiblement à droite et à gauche, ici pour faire ramasser des ordures qui devaient être protées dans une fosse, là pour organiser les corvées chargées d'aller chercher l'eau et le bois pour tout le monde. Ils avaient même un corps de garde, un lieu de réunion dans un bosquet de chênes. (Giono, 1977 : 436).

Pierre Citron, toujours dans la notice du Hussard, fait le rapprochement entre les charrettes où les morts sont entassés pendant l'épidémie dans le roman de Giono et l'amoncellement de cadavres des camps de Dachau, de Buchenwald et d'Auschwitz dont des photographies circulèrent à la fin de la Seconde Guerre frappant d'effroi et d'horreur ceux qui les virent et que ne pouvait que connaître l'écrivain de Manosque.

\section{米}

Les deux guerres mondiales sont essentielles pour comprendre Giono dans ses multiples contradictions, engagements, adhésions et refus, ainsi qu'elles demeurent une clé pour entrer dans son œuvre de fiction débarrassée de l'équivoque de sa couleur méridionale. Tant la Grande Guerre, décrite par Giono à ses vieux parents, que l'épidémie de choléra du Hussard sont en grande partie fabulées, sorties de son imaginaire en un mentir-vrai qui tient aussi bien du goût pour le conte qui édulcore les dangers des tranchées, dans le premier cas, que du goût de l'excès, dans le second. La maladie fonctionne comme un "réactif », terme employé par l'écrivain, qui révèle ce qui existe de meilleur et de pire en l'humain. L'exagération est également présente dans ses textes pamphlétaires sur la guerre afin de mieux convaincre son lecteur de l'importance d'une solution pacifique aux conflits évitant la surenchère militaire en France et en Europe dans les années 1930. Cinquante ans après sa mort, Jean Giono reste un écrivain de l'excès qu'il convient de lire sans modération. 


\section{BIBLIOGRAPHIE}

Album Giono (1980). Iconographie réunie et commentée par Henri Godard. Paris : Gallimard, « la Pléiade ».

BOISDEFFRE, Pierre de (1965). Giono. Paris : Gallimard, « bibliothèque idéale ».

CHONEZ, Claudine (1956). Giono. Paris : Seuil, « écrivains de toujours ».

CITRON, Pierre (1977). « Le Hussard sur le toit. Notice », in Jean Giono. CEuvres romanesques complètes IV. Édition établie par Robert Ricatte avec la collaboration de Pierre Citron et d'Henri Godard.

Paris : Gallimard, « la Pléiade », pp. 1305-1370.

CITRON, Pierre (1985). « Pacifisme, Révolte paysanne, Romanesque, sur Giono de 1934 à 1939 » in

Jean Giono, Imaginaire et écriture, Actes du colloque de Talloires (4, 5 et 6 juin 1984), Aix-en-

Provence : Édisud, pp. 25-44.

CITRON, Pierre (1986). « Légende et vérité, Giono pendant la guerre » in L'ARC 100, « Jean Giono », pp. 38-40.

GIONO, Jean (1971). CEuvres romanesques complètes I. Édition établie par Robert Ricatte avec la collaboration de Pierre Citron, d'Henri Godard, Lucien et Janine Miallet et Luce Ricatte. Paris : Gallimard, « la Pléiade ».

GIONO, Jean (1972). Euvres romanesques complètes II. Édition établie par Robert Ricatte avec la collaboration de Pierre Citron et de Luce Ricatte. Paris : Gallimard, « la Pléiade ».

GIONO, Jean (1974). CEuvres romanesques complètes III. Édition établie par Robert Ricatte avec la collaboration de Henri Godard, Lucien et Janine Maillet et Luce Ricatte. Paris : Gallimard, « la Pléiade ».

GIONO, Jean (1977). Euvres romanesques complètes IV. Édition établie par Robert Ricatte avec la collaboration de Pierre Citron et d'Henri Godard. Paris : Gallimard, « la Pléiade ».

GIONO, Jean (1989). Récits et essais. Édition publiée sous la direction de Pierre Citron avec la collaboration de Henri Godard, Violaine de Montmollin et Mireille Sacotte. Paris : Gallimard, « la Pléiade ».

GIONO, Jean (2015). Lettres de la Grande Guerre, 1915-1919. Revue Giono (Hors-série), Préface de Christian Morzewski, édition de Jacques Mény publiée par l'Association des Amis de Jean Giono.

HELLER-GOLDENBERG, Lucette (1972). Jean Giono et Le Contadour, « Un foyer de poésie vivante », 1935-1939. Nice - Paris : Publications de la Faculté des Lettres et des Sciences humaines de Nice - 9 / « Les Belles Lettres ».

LAMBERT, Emmanuelle (2019). Giono, furioso. Paris : Stock.

MAGNAN, Pierre (2002). Pour saluer Giono. Paris : Folio, ㄲo2448.

MÉNY, Jacques (1978). Jean Giono et le cinéma. Paris : Éditions Jean-Claude Simoën.

MORELLO, André-Alain (2008). « La guerre et la paix : Tolstoï, Alain, Giono », Association des Amis de Jean Giono, Revue Giono, ํ2, pp. 231-247.

THOMAS-MONTÉSINOS, Katia (2008). « Jean Giono et la guerre de 14-18: une expérience tragique et féconde », Association des Amis de Jean Giono, Revue Giono, n², pp. 183-208. 
TISSUT, Alain (2008). « Le miroir de la guerre », Association des Amis de Jean Giono, Revue Giono, no2, pp. 209-230.

\section{NOTES}

1. Dans Triomphe de la vie (1941) Giono écrit : «Tu t'étais efforcé de faire un Regain maigre. Pagnol en a tiré un film essoufflé, boursouflé et adipeux.» (Giono, 1989 : 660). À la fin de cet ouvrage, Giono imagine ce que pourrait être un scénario de Regain. Ce faisant on songe au différend qui opposa Marguerite Duras à Jean-Jacques Annaud quand il réalisa L'Amant qui aboutit à une réponse de la romancière sous forme d'un autre texte, L'Amant de la Chine du Nord.

2. Cette adaptation est désavouée par Giono qui poursuivra Pagnol en justice. Jean-Luc Godard, cité par Jacques Mény, à un mot très dur en comparant ce film et celui de François Villiers, fruit d'une collaboration directe avec Giono : "Qu'on ne me vienne pas me parler de Pagnol. La Femme du boulanger est à L'Eau vive ce que Jean Létraz est à Molière. » (Mény, 1979 : 57).

3. Ainsi l'article de Paris-presse - l'intransigeant, du 07/12/1954, intitulé « Giono siégera chez les Goncourt » qui affirme : « Mais à partir de 1947, Jean Giono reparait... Et stupéfie ses lecteurs. Il a renoncé à sa première manière; il ne vaticine plus sur la montagne ; il a abandonné Rousseau pour Balzac et Stendhal : Les Âmes forte, Le Hussard sur te toit, le moulin de Pologne, autant d'œuvres fortes, denses, qui sortent de la veine de La Chartreuse de Parme. » Dans Carrefour - la semaine en France et dans le monde, Claude Mauriac dans sa chronique "Les livres », du 16/02/1955, titré « La petite bibliothèque rouge » en parlant de romans récemment sortis parle du «nouveau Giono (celui du Hussard sur le toit)». Pierre de Boisdeffre dans son Giono, édité chez Gallimard dans la Collection La bibliothèque idéale en 1965 conçoit également l'existence de deux périodes, ce que l'écrivain de Manosque récuse absolument lors de l'émission la même année "La Minute de vérité » où il s'entretient à la radio avec Gilbert Ganne (rediffusé le 29/03/2020 dans le cadre d'une série en 10 épisodes proposée par Philippe Garbit sur France Culture). Giono insiste sur le fait qu'il n'y a pas rupture, mais continuité entre Que ma joie demeure et Le Hussard sur le toit. De façon paradoxale, le romancier constate « le bonheur » qu'il a pu éprouver en prison puisque Pour saluer Melville a justement été rédigé pendant sa première incarcération. Il constitue, toujours selon lui, le lien entre son roman de 1934 et celui de 1951.

4. L'attitude de Giono semble extrêmement contradictoire sous l'occupation. Il publie, entre le 3 décembre 1942 et le 18 mars 1943, Deux cavaliers de l'orage dans la Gerbe d'Alphonse de Châteaubriant, publication fondée en juillet 1940, antisémite, vichyste, préconisant le ralliement des catholiques et du monde agricole au nazisme. L'écrivain offre, en parallèle, refuge à Manosque à des juifs, communistes et réfractaires au Service du travail obligatoire (STO). Pierre Citron dans son texte "Légende et vérité, Giono pendant la guerre » réfute toute sympathie de l'écrivain envers le nazisme et le régime de Vichy et revient sur l'accueil qu'il donne à des réfugiés et son intervention en faveur de personnalités arrêtées comme le compositeur Jan Meyerowitz.

5. Correspondance réunie dans Jean Giono, Lettres de la Grande Guerre 1915-1919, édition établie, présentée et annotée par Jacques Mény, Revue Giono, Hors-série 2015, l'Association des Amis de Jean Giono, 2015.

6. Il reviendra sur ce détournement d'objet dans une autre lettre du 23 octobre : «Je rapporte un obus de 75 pour avion, superbe pot à fleurs» (Giono, 2015 : 172). On ne part plus à la guerre la fleur au fusil, on en revient avec des obus pot de fleurs.

7. Jean Giono, Le Grand Théâtre, «Bibliothèque de la Pléiade », Tome III, Paris, Gallimard, 1974. Le Grand théâtre, sorte de suite de Jean le Bleu, parait partiellement dans le numéro de mai 1961 de La Table ronde et fonctionne comme un hommage au père de l'écrivain, décrit comme une sorte de 
«Socrate provençal ». Euvre de commande pour accompagner une édition sous forme de livreobjet de l'Apocalypse de saint Jean.

8. Giono nourrit la plus vive admiration pour cet officier comme en témoigne la lettre à ses parents du 15 juin 1916: "J'ai une bonne nouvelle à vous apprendre: j'ai été attaché à la personne de notre capitaine. Notre capitaine est le meilleur homme que je connaisse : il est vieux, il a huit enfants et c'est un homme extraordinaire et très gentil. "

9. Ce message sera repris dans Les Cahiers du Contadour III-IV, achevé d'imprimer le 25 septembre 1937 et « dédié à la Grande Cause de la PAIX ». Il sera finalement repris en 1939 dans Précisions.

10. Après la Seconde Guerre, Giono dévalorise dans des lettres et lors d'entretiens ses rencontres avec des groupes de plus en plus nombreux (Heller-Goldenberg, 1972 : 12-13). Certains de ses participants étant à l'origine des Auberges de jeunesse, l'esprit « ajiste (goût pour la vie de plein air, mais également désir de promouvoir la fraternité entre les peuples et l'esprit de réconciliation entre anciens ennemis) détonne sur les Rencontres du Contadour qui se réalisèrent à Pâques et en septembre. C'est sans doute ce qui dire à Giono avec cet esprit de contradiction qui lui était commune à l'âge mûr qu'il ne s'agissait là que de « vacances ».

11. Antoine de Saint-Exupéry qui s'est lié d'amitié avec l'écrivain dédiera Le Petit Prince à «Léon Werth quand il était petit garçon ".

12. Dans la notice à Solitude de la pitié pour le premier volume des Euvres romanesques complètes publié dans la Bibliothèque de la Pléiade, Pierre Citron retrace la genèse de la nouvelle «Ivan Ivanovitch Kossiakoff » aux pages 1040 et 1041. Le romancier, après une conversation avec Lucien Jacques, écrit à ce dernier le 24 février 1925 : «Après votre départ je me suis mis à rédiger pour moi et pour vous l'histoire de Kossiakoff ".

13. Par exemple, la vision d'un cadavre d'enfançon comparé à un fromage blanc sera présente aussi bien dans Le Grand troupeau que dans Le Hussard sur le toit.

14. Jean Giono, Le Grand Troupeau, «Bibliothèque de la Pléiade », Tome I, Paris, Gallimard, 1971, p. 611.

15. «Le cinquième ange sonne de la trompette ", dans la deuxième partie, " Une grande étoile tomba sur les eaux » puis « ... Et la source des eaux devint amère ", dans la troisième partie.

16. Cité dans la note 1 .

17. Fuite à laquelle reste attaché le geste courageux du consul portugais Aristide de Sousa Mendes.

\section{RÉSUMÉS}

L'expérience de la guerre de 14-18 marque à jamais Jean Giono, mobilisé entre 1915 et 1919, gazé, bien que légèrement, meurtri par la disparition d'amis et de proches. De cette épreuve naît une profonde aversion à l'égard de la guerre et des discours patriotiques qui se traduira aussi bien par des écrits pacifistes dans l'entre-deux-guerres (Lettre aux paysans sur la pauvreté et la paix, Refus d'obéissance, Précisions) que par des textes de fiction comme la nouvelle de 1925, « Ivan Ivanovitch Kossiakoff » ou le roman de 1931, Le Grand troupeau. Publié en 1951, Le Hussard sur le toit, dont le thème principal est la traversée épique de la Provence en 1838 par un jeune hussard italien lors d'une épidémie de choléra, entretient une relation analogique étroite avec la guerre. Conflits et épidémies révélant ce qu'il y de meilleur et de pire en l'être humain fonctionnent comme de 
puissants réactifs qui inspireront l'œuvre de Jean Giono. C'est de ce parcours sous le signe de la guerre et de la paix dont il sera question dans cet article.

The experience of the war of 14-18 marks forever Jean Giono, mobilised between 1915 and 1919, gassed, although slightly, bruised by the disappearance of friends and relatives. From this ordeal arises a deep aversion to war and patriotic discourse, which will also be reflected in pacifist writing in the interwar period (Lettre aux paysans sur la pauvreté et la paix, Refus d'obéissance, Précisions) only through fictional texts such as the short story from 1925, "Ivan Ivanovitch Kossiakoff" or the 1931 novel, Le Grand troupeau. Published in 1951, Le Hussard sur le toit, whose main theme is the epic crossing of Provence in 1838 by a young Italian hussars during a cholera epidemic, maintains a close analogical relationship with the war. Conflicts and epidemics reveal the best and the worst in humans by functioning as powerful reagents that will inspire the work of Jean Giono. It is this journey under the sign of war and peace that will be discussed in this article.

INDEX

Mots-clés : Giono (Jean), littérature française (XXe siècle), première guerre mondiale, seconde guerre mondiale, épidémie dans la littérature (l')

Keywords : Giono (Jean), french literature (20th century), world war I, world war II, epidemic in literature (the)

\section{AUTEUR}

\section{LUIS CARLOS PIMENTA GONÇALVES}

Universidade Aberta

Luis.Goncalves[at]uab.pt 